\title{
Role of polyamines, their analogs and transglutaminases in biological and clinical perspectives
}

\author{
Enzo Agostinelli
}

Received: 22 August 2011/Accepted: 26 September 2011/Published online: 12 November 2011

(C) Springer-Verlag 2011

The ever growing interest in the biosynthesis and action of the ubiquitous polyamines with particular focus on the biology of normal and cancerous eukaryotic cells was the driving force to hold a 6-day Second international conference on the role of polyamines and their analogs in cancer and other diseases in Italy at SAPIENZA University of Rome and at the Grand Hotel Duca D'Este in Tivoli (Rome), December 1-6, 2010. The conference was organized by Prof. Enzo Agostinelli (SAPIENZA University of Rome) and Prof. Kazuei Igarashi (Chiba University, Japan). Interestingly, the bull of the Pope Bonifacio VIII established "SAPIENZA" on April 20, 1303. Apropos the conference in 2010, "SAPIENZA" celebrated its 707th anniversary. This Special Issue of Amino Acids brings together 62 peer-reviewed manuscripts that bring the essence of the lectures and posters presented at this conference. In addition, a few more papers on Polyamines and Transglutaminases were solicited from other experts who could not attend the meeting. These papers deal with highclass research data obtained until the mid-2011. ${ }^{1}$

Following the tradition of previously held successful congresses on polyamines worldwide, the Tivoli conference deliberated on several important issues related to the biochemical and pathophysiological properties of polyamines and transglutaminases as summarized by Bachrach (2011), in this issue. These papers project the essential role of polyamines not only in cell growth and differentiation in animals, plants and microrganisms but also in several

E. Agostinelli $(\bowtie)$

Istituto Pasteur-Fondazione Cenci Bolognetti,

Department of Biochemical Sciences, SAPIENZA University

of Rome and CNR, Institute Biology and Molecular Pathology,

Piazzale Aldo Moro 5, 00185 Rome, Italy

e-mail: enzo.agostinelli@uniroma1.it pathophysiological processes including carcinogenesis as well as in the development of new approaches to the therapy of cancer and other diseases. The polyamine biosynthetic pathway is very active during the growth of normally growing cells as well as in cancerous cells, polyamines often present at high concentrations. These increased levels occur because of enhanced synthesis of putrescine from ornithine catalyzed by ornithine decarboxylase (ODC), the rate-limiting enzyme, and also from increased uptake of polyamines (Marton and Pegg 1995). Major polyamines present in all eukaryotes include putrescine (diamine), spermidine (triamine) and spermine (tetraamine), and their derivatives, involve decarboxylation of ornithine, arginine and S-adenosylmethionine.

Modern history of polyamines dates back to 1958 when Tabor et al. (1958) first outlined their biological synthesis. Since then and, in particular, during the last 30 years, considerable progress has been made in understanding metabolic pathways for polyamines, enzymology involved, and genetic regulation. Polyamines being ubiquitous organic cations found in both eukaryotic and prokaryotic cells have attracted interest because of their multiple functions in cell biology (Pegg 1988; Cohen 1998; Agostinelli et al. 2010a) including, among many others, cell cycle regulation, gene expression and signal transduction (Bachrach et al. 2001; Childs et al. 2003; Mattoo et al. 2010).

Cellular polyamine concentrations are highly regulated. Their high intracellular accumulation, whether due to high extracellular concentrations or to deregulation of the intercellular systems that control polyamine homeostasis, can induce programmed cell death (apoptosis) in various cell types (Tobias and Kahana 1995; Pignatti et al. 2004;

\footnotetext{
${ }^{1}$ All manuscripts in this special issue were subjected to external peer reviewing according to the policy of this journal.
} 
Seiler and Raul 2005). Therefore, the involvement of polyamines in regulating cell proliferation and death has interested scientists to investigate their cellular function including mitochondria, where a range of cellular processes, such as energy production, proliferation, senescence and death occur (Goldenthal and Marin-Garcia 2004). Apparently, mitochondria lack a polyamine biosynthetic pathway; nevertheless, substantial quantities of spermine and spermidine have been detected in the mitochondrial matrix and a specific mitochondrial polyamine transporter has been described (Toninello et al. 2004).

In addition to being of vital importance for the viability and proliferation of most cells, polyamines spermidine and spermine are a source of cytotoxic metabolites. Polyamines are substrates of several flavin adenine dinucleotide (FAD)-dependent enzymes: monoamine oxidase (MAO), polyamine oxidase (PAO), spermine oxidase (SMO), and of copper amine oxidases (CuAOs): diamine oxidase (DAO), serum amine oxidases (SAO), lysyl oxidase (LOX) (Arancia et al. 2004; Agostinelli et al. 2004). The amine oxidases (AOs) oxidize polyamines and thereby regulate their intra- and extra-cellular levels. PAO is involved in the homeostatic regulation of polyamine pools; the other oxidases catabolize polyamines to ammonia and amino acids, which are then excreted via the kidneys (Seiler 1992). Polyamine oxidation products are toxic to cells. In serum-containing cell cultures, cytotoxicity of polyamines correlates with their property as substrates of serum amine oxidases (Morgan 1988). Mono-, di- and polyamines, as well as several $N$-acyl amines, are oxidatively deaminated by AOs in the presence of $\mathrm{O}_{2}$ and $\mathrm{H}_{2} \mathrm{O}$. The possibility of using purified bovine serum amino oxidase (BSAO) in the presence of exogenous spermine or endogenous polyamines to induce cytotoxicity in several human tumor cell lines in vitro (Calcabrini et al. 2002; Agostinelli et al. 2009) or injecting the enzyme into the tumor in vivo (Averill-Bates et al. 2005) has been described. Amine oxidases metabolize preferentially polyamines (spermine and spermidine) to generate the reaction products $\mathrm{H}_{2} \mathrm{O}_{2}$ and aldehyde(s) (Agostinelli and Seiler 2006). Such toxic products are able to induce stress-activated signal transduction pathways, leading to cell death, by necrosis or apoptosis (Lindsay and Wallace 1999; Seiler and Raul 2005; Agostinelli et al. 2006).

Post-translational modifications of proteins via covalent binding of polyamines with special emphasis on transglutaminases (TGases) and hypusine biosynthesis are also highlighted in this issue. TGases are ubiquitous enzymes with multifaceted functions. Current research on TGases includes both basic and applied aspects, involving the fields of medicine (e.g., development of diseases such as cancer and Alzheimer), plant science (e.g., regulation of processes, such as photosynthesis and fertilization), and food and material sciences (production of protein based materials and cross-linked proteins as binders). TGases posttranslationally modify proteins by a number of methods one of which includes the incorporation of polyamines through peptide-bound glutamine with potential in cancer and other TGases-related diseases. As competitive primary amine substrate of TGases, polyamines can also act as physiological inhibitors of protein cross-linking. By utilizing this property, when conjugated to biotin or FITC they can be used as physiological probes, enabling detection and monitoring of the active enzyme both at the cell surface and in the extracellular matrix under both physiological and pathological conditions thus helping to elucidate the many functions of TGases.

This issue is intended to provide a relatively short overview of some important concepts and aspects of polyamines and TGases that provide a timely guidance for researchers in this interdisciplinary field encompassing the domains of pharmacology, molecular biology, physiology, biochemistry, biotechnology and cancer research. The first part of the issue starts with articles entirely devoted to polyamine catabolism and its role in animals and plants. This information could be useful in evaluating the possibility of this metabolic pathway as a target for new antiproliferative therapies in animals and stress tolerance strategies in plants (Tavladoraki et al. 2011). Alterations in polyamine metabolism have been linked to a variety of disease states, most prominently to hyperproliferative diseases such as cancer (Bachrach 2004). A study of two different transgenic mouse lines with enhanced polyamine catabolism has uncovered a new link between polyamine metabolism and metabolic diseases, such as obesity or diabetes (Cerrada-Gimenez et al. 2010). Koponen et al. (2011) demonstrate that by enhancing the polyamine catabolism in the white adipose tissue, the transgenic mice have reduced white adipose tissue and are protected against the negative effects of a high fat diet. To achieve improved glucose tolerance, it may be a good idea to enhance polyamine catabolism in liver and skeletal muscle, in parallel with white adipose tissue.

Spermine oxidase (SMO) specifically oxidizes spermine (Spm) and plays a dominant role in the highly regulated mammalian polyamines catabolism. SMO dysregulation alters polyamine homeostasis participating in the etiology of several pathological conditions, including cancer (Amendola et al 2009; Agostinelli et al. 2010b; Casero and Pegg 2010). Direct mechanistic links among inflammation, SMO activity, ROS production, and carcinogenesis have been demondstrated (Goodwin et al. 2008). The review article by Cervelli et al. (2011) highlights the main biochemical, cellular and physiological processes in which SMO is involved. 
The review article by Chaturvedi et al. (2011) summarizes the importance of utilizing L-arginine as a host response to the gastric pathogen Helicobacter pylori. The authors show that generation of antimicrobial nitric oxide (NO) by inducible NO synthase (iNOS), which is dependent on L-arginine availability, is limited by competition with arginase II activity (Lewis et al. 2011) and upregulation of ornithine decarboxylase. Spermine blocks L-arginine uptake into macrophages (Chaturvedi et al. 2010) and SMO mediates DNA damage in gastric epithelial cells.

Another aspect of polyamine metabolism covered in this issue deals with spermidine/spermine $N^{1}$-acetyltransferase (SSAT1), a key enzyme catalyzing the $N$-acetylation of spermine and spermidine. SSAT1 activity is regulated at several levels, e.g., transcription, pre-mRNA splicing, translation, and protein stabilization. Increased expression of SSAT1 alters polyamine levels, increases oxidative damage, or decreases availability of acetyl-CoA and ATP, which affect physiological and patho-physiological processes such as carbohydrate and lipid metabolism, pancreatitis, ischemic damage and carcinogenesis (Pegg 2008). Studies by the Finnish group (Uimari et al. 2011; Pirnes-Karhu et al. 2011; Hyvönen et al. 2011a), reported in this issue, now show that polyamines regulate the alternative splicing of SSAT1 in vivo, contributing to fine-tuning of SSAT1 expression. In addition, the overexpression of SSAT1 in mice seems to enhance acute anti-inflammatory response phase induced by LPS, while SSAT1 overexpression and spermine analog, $N^{1}$ $N^{11}$-diethylnorspermine treatment, attenuate the severity of zinc-induced pancreatitis.

Spermidine synthase (SpdSyn) is an aminopropyl transferase (reviewed in Ikeguchi et al. 2006) that converts the diamine putrescine to the polyamine spermidine, which has implications in normal cellular function as well as disease (Eisenberg et al. 2009; Park et al. 2010). In this regard, the article by Shi et al. (2011) shows SpdSynoverexpressing transgenic mice are viable and exhibit reduced tissue spermine:spermidine ratios. Concurrent transgene-driven overexpression of SpdSyn along with either spermine synthase or $S$-adenosylmethionine decarboxylase did not dramatically alter polyamine accumulation or reduce viability. Therefore, SpdSyn activity does not seem to be a primary regulator of basal polyamine content or it being rate-limiting for polyamine biosynthesis.

A part of the issue is extensively devoted to the enzymology and molecular biology of ornithine decarboxylase (ODC). This enzyme is a rate-limiting step in polyamine biosynthesis and associates with a specific antizyme except for plants. ODC antizyme inhibitors act mainly as positive regulators of polyamine levels by binding to antizymes (Kahana 2009; Mangold 2006; López-Contreras et al 2010). There is also evidence that they may affect other important cellular proteins (Kahana 2009; Dulloo et al.
2010). In mammals, there are three antizymes (AZ1, AZ2 and AZ3) and two antizyme inhibitors (AZIN1 and AZIN2). Ramos-Molina et al. (2011) report the relative expression levels of all these genes in different rodent tissues and human cell lines in an attempt to clarify their physiological role. ODC AZ1 is a translationally controlled, regulatory protein that binds to ODC and targets it for ubiquitin-independent proteosomal degradation (Kahana 2007). Fraser et al. (2011) provide proof-of-principle concept that knockdown of AZ1 results in increased sensitivity to the antitumor analogue, $N^{1}, N^{11}$-bis(ethyl)norspermine. On a different note, it seems unlikely that the antizymes with their complex regulation have only evolved to regulate polyamines. Indeed, recent studies have shown that AZ1 regulates several growth related proteins other than ODC (Dulloo et al. 2010). Pietilä et al. (2011) present data that swift repression of $\mathrm{LNCaP}$ prostate cancer cell proliferation after conditional AZ1 induction was not accompanied by depletion of polyamines. Ishii et al. (2011a, b) show that vascular smooth muscle cells cultured in three-dimensional matrix, called honeycombs, exhibit lower proliferation rates through decreasing polyamine concentration and FAK phosphorylation at a high level of AZ1. Subsequently, these cells increased the levels of $\alpha$ actin and myosin for differentiation. Giordano et al. (2011) studied the cooperation between cardiac polyamines and Larginine (L-Arg) availability in transgenic mice overexpressing ODC in the heart (MHC-ODC mice). These animals experienced a much more dramatic left ventricular hypertrophy in response to beta-adrenergic stimulation with isoproterenol (ISO) compared to non-transgenic controls (Shantz and Giordano 2006). MHC-ODC mice administered L-Arg along with ISO showed a rapid onset of systolic dysfunction and died within $48 \mathrm{~h}$. The induction of arginase observed in this setting is thus presented as a protective response to beta-adrenergic stimulation in the presence of high levels of polyamines. Antizyme inhibitors (AZI/AZIN) are key regulators of the polyamine pathway. AZIs interfere with antizyme functions, leading to increased polyamine biosynthesis, increased cell proliferation, as well as enhanced cell transformation and tumorigenesis (Mangold 2006; Keren-Paz et al. 2006). AZIs are overexpressed in several human tumor types and recent studies, including those of Olsen et al. (2011), in this issue, suggest that interfering with AZI function may decrease tumor growth. The authors, in their study, made a series of prostate cancer cell lines stably expressing shRNA (short hairpin RNA) to knockdown antizyme inhibitor 1 (AZI) or control shRNA. Their results demonstrate that suppression of AZI levels in prostate cancer cell lines results in repression of cell proliferation and tumor growth in vivo. These results suggest that in vivo suppression of AZI presents a potential target for anti-tumor therapy. 
The ODC activity of plants is tightly correlated with rates of cell proliferation and is also highly inducible by wounding and other signals. Metazoan and fungal ODCs are negatively feedback regulated in response to excess polyamines by producing the inhibitory antizyme. Illingworth and Michael (2011) sought to determine the identity of plant ODC-binding proteins and found that only ribosomal protein S15 bound to a plant ODC. Delicate balance between translation and degradation of ODC, ODC antizyme, SSAT and S-adenosylmethionine decarboxylase (SAMdc) tightly controls the intracellular polyamine levels (Perez-Leal and Merali 2011).

A previous model of mammalian polyamine metabolism showed that SAMdc activity could play a key role in polyamine homeostasis (Rodríguez-Caso et al. 2006) as has been confirmed for plants (Mattoo et al. 2010). In this issue, Reyes-Palomares et al. (2011) show a combined model that integrates the previously published polyamine model (hepatic polyamine) and one-carbon and glutathione metabolism model, is able to reproduce the predictions of each individual model. A transition between two versions of the combined model with additional regulatory factors simulates properly the switch in methionine adenosyltransferase isozymes occurring when liver enters proliferative phase. In fact, the first mathematical model of polyamine metabolism in mammalian cells predicted that S-adenosylmethionine/Met availability could be the major regulatory factor of polyamine levels (Rodríguez-Caso et al. 2006). Correa-Fiz et al. (2011) show that mice liver administrated $0.5 \%$ Met (in drinking water for 5 months) indeed have an increased level of spermidine. Polyamine metabolism including acetylation and oxidation process play various physiological roles, for example, as markers such as acrolein for stroke and renal failure (Saiki et al. 2011). Another function of polyamine metabolism presented here (Ishii et al. 2011a) is in adipogenesis of 3T3-L1 cells.

Polyamines are involved in other cellular responses, such as differentiation, apoptosis and hypertrophy. Marverti et al. (2011) report that distamycin A and its two synthetic analogues differently affect expression in vivo of folate cycle enzymes in a cisplatin-sensitive human ovarian cancer cell line and their resistant counterpart, which also showed cross-resistance to some polyamine analogs (Marverti et al. 2010). The results also show that these poly-pyrrolamidine derivatives modulate the cell growth effects of cisplatin and a polyamine analog through the induction of the polyamine catabolic pathway. An up-regulation of SSAT caused by this compound was observed. Arisan et al. (2011) focus on the role of roscovitine, a purine derivative cyclin-dependent kinase (CDK) inhibitor, which strongly induces apoptosis by altering mitochondrial apoptotic pathway. The depletion of total polyamine content by the treatment of $\alpha$-difluoromethylornitine (DFMO), an ODC inhibitor increased the roscovitine-induced apoptosis by altering several $\mathrm{Bcl}-2$ family members in HCT116 cells. In addition, roscovitine up-regulated SSAT expression and caused a significant increase in reactive oxygen species. These authors conclude that polyamine metabolism might play a critical role in cells death by apoptosis.

A link between heart hypertrophy and polyamines was previously established which implicated polyamines in the development of cardiac diseases (Cetrullo et al. 2010). Facchini et al. (2011), in this issue, provide evidence that polyamines may favor hypertrophy and terminal differentiation of articular chondrocytes, raising the possibility that the polyamine pathway represents a potential target to control inappropriate chondrocyte maturation in osteoarthritis.

Understanding the role of highly conserved proteins across evolution, like those involved in protein synthesis, is essential not only to establish the basis of the mechanisms involved in the regulation of gene expression, but also to help modulate such processes during disease conditions. Eukaryotic initiation factor 5A (eIF5A) is one of these proteins and constitutes an interesting drug target. eIF5A undergoes a unique posttranslational modification to form an unusual amino acid hypusine, whereby spermidine is used to convert lysine residue to hypusine, which is essential for eIF5A activity and cell proliferation (Park 2006; Park et al. 2010; Zanelli and Valentini 2007). Several contributions in this issue deal with this topic. Nishimura et al. (2011) demonstrate that eIF5A-1 and deoxyhypusine synthase genes are essential for mouse embryonic development. Hyvönen et al. (2011b) show that only those C-methylated spermidines that act as substrate for hypusine synthesis substitute natural spermidine in supporting cell growth. The finding reinforces the notion that hypusine synthesis is a key function of polyamines in cell proliferation. Dias et al. (2011) used the yeast model and genetic analysis to confirm a role for eIF5A in translation elongation. It was demonstrated that eIF5A functionally interacts with an elongation factor 2 (eEF2) in the cell. Kaiser (2011) reviews some functions of eIF5A. Revisiting an old acquaintance, eIF5A also has a nuclear membrane export protein function as an mRNA-protein shuttle (Hauber 2010). Thus, eIF5A plays a role in the pathogenesis of different diseases such as diabetes, some human cancers, malaria and HIV-1 infections.

The importance of the trans-membrane transport to polyamine homeostasis has long been recognized. Indeed, the import of exogenous polyamines from nutritional as well as microflora origins provides a major fraction of the total needs for polyamines in mammals. However, the identity of the mammalian entities responsible for polyamine uptake still remains elusive. Poulin et al. (2011) summarize the current state of knowledge in this area and 
update the recent promising advances in identifying metazoan polyamine permeases. Tomitori et al. (2011) present an interesting review on the structure and function of polyamine-amino acid antiporters, CadB and PotE, in Escherichia coli. $\mathrm{CadB}$ is a cadaverine-lysine antiporter and PotE is a putrescine-ornithine antiporter in E. coli (Soksawatmaekhin et al. 2006; Kashiwagi et al. 1997, 2000). A structural model of $\mathrm{CadB}$ and PotE is shown in this paper. The central cavity of $\mathrm{CadB}$, containing the substrate-binding site, is wider than that of PotE, mirroring the size differences of cadaverine and putrescine. Agostinelli et al. (2011) present a paper on this aspect. Polyamine transport across the mitochondria membrane occurs by a specific, common uniporter system and appears controlled by electrostatic interactions (Stevanato et al. 1994) as found for polyamine oxidative deamination by AOs. In virtue of the structure-function relationship of proteins, a similar structural topology can be invoked for the polyamine-binding domains of the AOs. Such a common structure may originate from an ancestral polyamine-recognizing gene.

Observations on the interactions of biogenic amines with mitochondria reveal new intriguing mechanisms of amine oxidation and transport previously investigated (Agostinelli et al. 2004, 2010b; Battaglia et al. 2010). Grancara et al. (2011) present data on the presence of two different mechanisms on the effects of monoamine oxidation on liver and brain mitochondria in inducing the permeability transition (MPT). In liver, the phenomenon is strictly related to an oxidative stress while in brain a different, as yet unknown mechanism exists. Similarly, liver and brain mitochondria differ in the regulation of spermine transport (Battaglia et al. 2011). In liver, spermine uptake is regulated by the redox state of mitochondria, while in the brain the regulation involves tyrosine phosphorylation. Martinis et al. (2011) show that agmatine transport is regulated by the binding of this amine to the imidazoline receptor $\mathrm{I}_{2}$, thus giving it a peculiar physiological role unknown until now.

Agmatine, is an endogenous polyamine metabolite formed by decarboxylation of L-arginine ( $\mathrm{Li}$ et al. 1994). Many studies have demonstrated a possible involvement of agmatine in oxygen consumption, ATP synthesis and mitochondrial membrane potential. In fact, experimental data give evidence for multiple molecular targets of agmatine with no toxic effects. Dependent on the guanidino group, agmatine shows a strong basic character, differing from other monoamines in that it carries one or two positive charges at physiological pH. Many observations also confirm agmatine in the brain may have neuroprotective effects (Reis and Regunathan 2000). Condello et al. (2011a) previously demonstrated that agmatine administration preserves mitochondrial membrane potential $\left(\Delta \psi_{\mathrm{m}}\right)$, thereby counteracting rotenone-induced cell damage in neuronallike differentiated SH-SY5Y neuroblastoma cells. Now, Condello et al. (2011b) present biochemical and structural changes resulting from rotenone-induced cell stress and the protective effects of agmatine in this model of Parkinson's disease (PD), using Fourier transform infrared (FTIR) spectroscopy. Based on studies by Satriano et al. (1998), in which agmatine was shown to act as an antiproliferative molecule and a potential tumor suppressor by restricting the availability of cellular polyamines, Bandino et al. (2011) exposed rat liver hepatoma cells (HTC) to increasing agmatine concentrations over a long term (i.e., several months) and found a clone of agmatine resistant (Agres) cells. These Agres cells showed that agmatine-mediated antiproliferative effects were related to deficiency of polyamine transport.

The role of polyamines in differentiation (Pignatti et al. 2004) was investigated by Compagnone et al. (2011), using epithelial to mesenchymal transition (EMT) and mesenchymal to epithelial transition (MET) in epithelial phenotype MDCK cells (Kalluri and Weinberg 2009). Intracellular levels of polyamines decreased during EMT induced by TGF- $\beta 1$, whereas addition of exogenous polyamines reverted it to epithelial phenotype cells. Low intracellular level of polyamines favored nuclear accumulation of poly-sumoylated transcription factor Snai1, a "zinc-finger protein".

Ornithine decarboxylase (ODC) and SAMdc are ratelimiting enzymes in polyamine biosynthesis (Igarashi and Kashiwagi 2010). ODC mRNA was at a relatively high level at the beginning of oogenesis of Xenopus. Mishina et al. (2011) show that polyamines modify the mRNA of morphogenetic transcription factor beta-catenine, which induces a secondary head formation in early embryogenesis of Xenopus.

New data on the metabolic peculiarities of parasites with respect to glutathione and trypanothione (bis-glutathionyl spermidine) metabolism are reported in Leishmania. Leishmania parasites possess a unique polyamine-based redox metabolism, in which trypanothione replaces many of the antioxidant functions of glutathione in mammals (Colotti and Ilari 2011). Ilari et al. (2011) solved the crystal structure of Leishmania infantum trypanothione reductase (TR) in complex with $\mathrm{Sb}(\mathrm{III}), \mathrm{Ag}(\mathrm{I})$ and with auranofin, an anti-rheumatic organogold drug. They show that other metal-based compounds other than antimonials inhibit TR by binding to the catalytic cysteines, thereby, killing the parasite (Baiocco et al. 2011). These compounds may lead to the development of new therapeutic strategies against leishmaniasis.

Apart from the developments in the polyamine research using animal and parasite models, research in recent years on understanding the biosynthesis, catabolism, and action 
of polyamines in plants have brought in new insights to bear on the field. This progress has been largely due to the change in approach, graduating from pharmacological approaches to applying molecular genetics tools to produce gene knockouts (mutants) and gain-of-function transgenic plants (Moschou et al. 2008). Thus, in plants, recent discoveries of spermidine and/or spermine function include their role as metabolic memory rejuvenators (Mattoo and Handa 2008), prolongers of life span of vegetative and fruit tissue-coined 'longevity elixir', stress relievers, and defense molecules against pathogens. Importantly, polyamines' nexus with cellular metabolism has been attracting more and more attention, the effects of diamine putrescine being in contrast to those of spermidine and spermine (Mattoo et al. 2010). Amino acids and polyamines influence each other's cellular concentration, and quantifying their cellular levels has been coined the term 'aminome' in a paper by Kausch et al. (2011), in this special issue. This article demonstrates that co-suppression of lipoxygenase (LOX) in tomato fruit leads to the deficiency of the plant hormone methyl jasmonate (MeJAS), which is associated with decreased levels of polyamines and 16 out of the 30 metabolites examined including 12 amino acids and GABA. The findings of Kausch et al. may be relevant and provide some clue to the earlier observations showing MeJAS treatment can induce death of lymphoblastic leukemia cells as well as several human cancer cell lines (Fingrut and Flescher 2002).

As in animals, spermidine and spermine are synthesized in plants by aminopropyltransferases (APTs) that transfer aminopropyl moieties, derived from decarboxylated S-adenosylmethionine, to putrescine and spermidine to produce spermidine and spermine, respectively. The minireview by Shao et al. (2011) complements several recent reviews on the overall biosynthetic pathway of polyamines and their physiological roles in plants and animals (Handa and Mattoo 2010; Igarashi and Kashiwagi 2010). Moreover, it compares plant APTs with animal and microbial enzymes and points out possible future applications of plant APTs for the improvement in nutritional value and stress responses of plants.

In plants, polyamines play pivotal roles in a wide range of growth, developmental and defense processes (Kusano et al. 2008; Handa and Mattoo 2010). Indeed, changes in polyamine levels occur during certain plant developmental transitions or exposure to stress conditions. In addition, exogenous applications of polyamines have frequently been shown to affect plant growth and response to various biotic and abiotic stresses. Loss-of-function mutations in polyamine metabolism genes have also evidenced the importance of polyamines in plant development and stress tolerance. The comparative data presented by Fincato et al. (2011b), in the present issue, on the tissue- and organ- specific expression pattern of the Arabidopsis thaliana PAOs, enzymes involved in polyamine back-conversion (Tavladoraki et al. 2006; Fincato et al. 2011a), will contribute greatly to better understand the physiological roles of polyamines and of their metabolic pathways in plant growth and development. In Arabidopsis thaliana, a model plant, polyamine catabolic pathway was unveiled (Fincato et al. 2011a). A. thaliana contains 5 PAO-encoding genes and, of them, $4 P A O$ s are involved in back-conversion but not in terminal catabolism. Ono et al. (2011) report that three PAOs out of 7 in Oryza sativa, a monocotyledonous model plant, localize in peroxisome and catalyze polyamine back-conversion reactions.

Due to their chemical attributes, polyamines can interact with different classes of macromolecules, alter structural states and influence metabolic activity by modifying transcription or translation events. On this basis, polyamine research is an important field for drug development with great potential for identifying new molecules for different diseases. The identification of the targets is a first step for developing strategies to elucidate the mechanisms involved. Previously, Groppa et al. (2008) had studied the relationship between polyamines and nitric oxide (NO) in connection with wheat root growth and the regulation of nitrate reductase (NR) by NO in wheat leaves (Rosales et al. 2011a). In this issue, Rosales et al. (2011b) have assessed the involvement of $\mathrm{NO}$ and polyamines in regulating NR activity in wheat leaves segments.

Previously, polyamine analogs were designed, synthesized and tested as anticancer agents (Casero and Woster 2009) as well as in neurodegenerative processes (Minarini et al. 2010), antiparasitic compounds, neurotransmitter receptor neuroprotectants, and multitarget-directed ligands for multifactorial diseases. Some polyamine analogs have now been found to be also good "skeletons" for the development of novel inhibitors of semicarbazide-sensitive amine oxidase/vascular adhesion protein-1 (SSAO/VAP-1) and of monoamine oxidase $\mathrm{B}$ (MAO B). Bonaiuto et al. (2011) evaluate in this issue test some synthetic polyamines as inhibitors of two new potential targets, human SSAO/VAP-1 and MAO B, enzymes involved in various multi-factorial diseases such as Alzheimer's disease.

Dysregulation of epigenetic control of gene expression is a common feature in the initiation and progression of cancer. DNA methylation and covalent modification of histones collaborate to function as epigenetic regulators of gene expression. Lysine specific demethylase 1 (LSD1) is a chromatin-modifying enzyme that can broadly repress transcription by demethylating the transcriptional activating mark of dimethyl lysine 4 of histone 3 (H3K4) in the promoters of important tumor suppressor genes. Because of the similarity in structure and enzymatic activity between LSD1 and the PAOs, SMO and $N^{l}$-acetylpolyamine 
oxidase (APAO), it was hypothesized that specific polyamine analogues may act as effective inhibitors of LSD1 and induce the re-expression of aberrantly silenced genes in cancer. Zhu et al. (2011) have now demonstrated both in vitro and in vivo that the strategy of using several polyamine analogues to inhibit LSD1 does lead to re-expression of aberrantly silenced tumor suppressor genes resulting in effective antitumor effects that merit clinical investigation. In this regard, Nayvelt et al. (2011) report an unusual activation of estrogen responsive genes and increased DNA synthesis in the presence of a bis(ethyl) polyamine analogue. These intriguing results suggest that membrane localized or nuclear estrogen receptor may be activated by cell signaling perturbations caused by this polyamine analogue (Thomas and Thomas 2001). Greater attention to the heterogeneity of cellular responses, based on their stage of growth or cell cycle phase and the differentiation of normal and cancer cells, might facilitate drug discovery process by selectively obliterating tumor cells. It was observed that the breast cancer cell line $\mathrm{L} 56 \mathrm{Br}-\mathrm{C} 1$ does not express the cell cycle regulatory retinoblastoma protein (pRb). Treatment with the polyamine analogue $N^{1}, N^{11}$ diethylnorspermine (DENSPM) causes apoptosis in L56BrC1 (Hegardt et al. 2002). Now, Johansson et al. (2011) show that overexpression of $\mathrm{pRb}$ rescues DENSPM-treated L56Br-C1 cells while lowering the activity of the polyamine catabolic enzyme SSAT. This suggests that the $\mathrm{pRb}$ protein may be a marker for polyamine depletion sensitivity and that there is a connection between $\mathrm{pRb}$ and the regulation of SSAT activity.

To conclude the first part of this editorial, two papers in this issue describe new methods to determine polyamine flux in cell culture or biogenic amines (BAs) in infusions of black and green tea. Earlier, radiolabeled ${ }^{3} \mathrm{H}$ or ${ }^{14} \mathrm{C}$ polyamines or their amino acid precursors in addition to synthetic non-natural precursors, like 2-fluoro-ornithine, were used to monitor polyamine flux (Kramer et al. 2008). Both the previously developed methods have built-in limitations hampering their general use for flux analysis. CerradaGimenez et al. (2011) have developed a novel LC-MS/MS method for determining polyamine flux that is based on label incorporation from the ${ }^{13} \mathrm{C},{ }^{15} \mathrm{~N}$-labeled amino acid precursors into polyamines (Häkkinen 2011). This method is applicable for studying polyamine flux in cell cultures and overcomes the limitation associated with the previous flux analysis methods. Like in all plants, dried leaves and buds of tea plant Camellia sinensis contain BAs and polyamines. Brückner et al. (2011) have quantified BAs in infusions of black and green tea, commonly consumed as beverages, using HPLC and derivatization with Fmoc-Cl. Since no histamine was detected, and polyamines and tyramine were present at very low concentrations ( $\mu \mathrm{g}$ range), no health risks related to BAs are to be expected even after drinking very large quantities of tea. These data add to the databases for BAs and polyamines from fermented foods and beverages (Kirschbaum et al. 2000).

The second part of this issue is dedicated to novel aspects of TGases related to neoplastic diseases and in the pathogenesis of celiac disease. In addition, studies on the applications of TGases in material science and as potential biotechnological tools are included. TGases were first described as enzymes facilitating the formation of $N-\varepsilon$ ( $\gamma$-glutamyl)lysine isopeptide bond, when it takes place between endo- $\gamma$-glutaminyl and endo- $\varepsilon$-lysyl protein residues, or the covalent incorporation of diamines and polyamines into proteins (Folk 1983). Dependent on the ability to produce protein cross-links, TGases have been referred to as "Nature's Biological Glues" (Griffin et al. 2002). Alternatively, the deamidation of specific glutamine residues in the absence of suitable amine acceptors can also occur. In this regard, the involvement tissue TGase 2 has become highly significant in the pathogenesis of celiac disease (Sollid and Khosla 2005). Increased amounts of TGase 2 can be involved in certain types of cancer. In particular, TGase 2 seems involved in antiapoptotic as well as proapoptotic function depending on the levels of intracellular calcium. TGases have the potential as biotechnological tools because they can be useful outside of their natural environment (Di Pierro et al. 2010). Given the accumulating evidence for the physiological and pathological importance of extracellular tissue TGase2, the review article by Wang and Griffin (2011) summarizes the roles of the enzyme both as a novel cell adhesion protein and through its interaction with cell surface Syndecan 4 (Wang et al. 2010). As an enzyme capable of modifying proteins either through crosslinking, deamidation or via the incorporation of primary amines like polyamines, the importance of TGase 2 both at the cell surface and in the extracellular matrix has been assessed. The review also outlines the importance of TGase 2 in diseases like cancer, celiac disease, and tissue fibrosis (Iismaa et al. 2009). Therefore, TGase2 is not only an important player in many pathological processes, but it is also a potential bio-marker and novel therapeutic target for their treatment. In celiac disease, TGase 2 is specifically involved in disease pathogenesis by first deamidating gliadin peptides, which induce a more pronounced T-cell response, and secondly, as being target of IgA class autoantibodies (Caputo et al. 2009; Lindfors et al. 2009). Teesalu et al. (2011), in this issue, suggest that antiTGase 2 antibodies from patients inhibit TGase2 binding to heparin/heparan sulfate and, consequently, can affect cell adhesion function of TGase2. Further research has to demonstrate whether a proposed function of autoantibodies has a role in the immunopathogenesis of the disease. 
Caccamo et al. (2011) review the role played by TGase 2 in cell response to redox state imbalance both under physiological and pathological conditions, such as neurodegenerative disorders, inflammation, autoimmune diseases, and cataractogenesis, in which oxidative stress plays a pathogenic role and also accelerates disease progression. The knowledge that very different physiological and pathological processes are dependent on the presence of adequate levels of these cross-linking enzymes and on the amount of both free and protein-conjugated polyamines by TGases is the subject of Tabolacci et al. (2011). TGases are increasingly recognized as important players in the vascular system. Thus, TGase2 and FXIII-dependent crosslinking of matrix elements within the vascular wall was found to be involved in physical remodeling of small arteries (van den Akker et al. 2010) and arterial calcification. Throughout the TGase field, it has remained an enigma how TGase 2 is secreted, and the vascular system is no exception to this. Now, van den Akker et al. (2011a) provide evidence that TGase 2 is secreted from vascular smooth muscle cells by transamidation-dependent microparticle formation. Together with the recent work on the redox balance in TGase 2 activation (van den Akker et al. 2011b), novel insight into the vascular actions of TGase 2 are gained that open ways for pharmacological interference in cardiovascular diseases. Among the TGases, transglutaminase 4 (TGM4), a prostate-specific enzyme found in most mammals, is generally believed to be responsible for the formation of copulatory plug, which is a common means of reducing competition with rival males. Tseng et al. (2011) reveal a special preference of mouse TGM4 to the seminal proteins that are disulfide cross-linked beforehand. Such kind of mutual adaptation between mouse TGM4 and its native substrates enhances not only the sperm competition of male mice but also the perspective on relationship between TGases and their substrates.

Comprehensive analysis of the functional role of individual TGases in rodent models has been limited largely due to compensatory effects in knockout mice. In addition, identification of new therapeutics targeting TGases in disease with animal models is very expensive and under rigorous ethical supervision. Therefore, zebrafish was evaluated as a novel biological model for in vivo studies of TGases (Deasey et al. 2011), in this issue. The TGase gene family in zebrafish (Danio rerio) has been characterized for the first time and functional investigations provide the long lacking in vivo evidence for TGase-mediated regulation of bone development (Nurminskaya et al. 2003; Deasey et al. 2011).

Transglutaminases are attractive enzymes for biotechnology due to their ability to stabilize scaffolds via crosslinking. However, these enzymes may also affect cell behavior and differentiation programs. Regulation of chondrogenic differentiation in human bone-marrow derived stem cells was studied on scaffolds of Collagen type XI. This collagen is deposited by mesenchymal stem cells undergoing chondrogenic differentiation (Xu et al. 2008) and has been identified as a potential substrate for tissue TGases by $\left[{ }^{3} \mathrm{H}\right]$-putrescine incorporation (Kleman et al. 1995). Shanmugasundaram et al. (2011) show that TGase2-mediated crosslinking of collagen XI accompanied by autocross-linking of TGase 2 protein promotes chondrogenesis in human stem cells. This study suggests an attractive alternative for enhanced cartilage formation through TGase2-mediated scaffold modifications versus genetic alteration of the cells. Applications of TGases in material science require optimized enzymes, for instance with higher thermostability, as addressed by Büttner et al. (2011) in this issue. These enzymes can be overproduced and purified on a mass scale as described by Sommer et al. (2011).

Besides representing widely distributed enzymes with pleiotropic functions in animals, TGases have important roles in plant science. TGases have been detected in algae and angiosperms; the main TGase reaction studied in plants has been the post-translational modification of proteins by the conjugation of polyamines. Possible roles concern the stabilization of the photosynthetic complexes and Rubisco, the modification of cytoskeleton proteins, and the involvement in the cell wall organization. Other roles appear to be related to fertilization, abiotic and biotic stresses, programmed cell death and pollen allergy (Serafini-Fracassini et al. 2009; Del Duca et al. 2010). Gentile et al. (2011) analyze the role of TGases and polyamines in the progammatic phase of plant reproduction, showing that polyamines and TGases are involved in pollen-pistil interactions during the self-incompatibility response. Moreover, in pollen, TGase plays a possible role in the modification of cytoskeleton proteins during apical growth. The report that climatic changes influence pollen allergenicity (Cecchi et al. 2010) led Iorio et al. (2011) to investigate the effects when pollen is subjected to simulated stressful conditions (changes in temperature, humidity, copper and acid rain pollution). It was found that TGase activity increased under stressful treatments and, along with its products, was released outside the pollen. The authors hypothesize that externalized pollen TGase could be one of the mediators of pollen allergenicity, possibly by stimulating the phospholipase activity, especially under environmental stress induced by climate changes.

In conclusion, the interest in polyamine and TGase function has continued with a new crop of researchers, who have replaced several authoritative scientists in this field who have sadly passed way. The foundation laid by the previous scientists will remain a cornerstone for the new 
generation of scientists to reveal still new and unexplored facets of the function of polyamines.

Acknowledgments The editors wish to thank all the participants and institutions for their kind contribution to the 2nd International Conference on the Role of Polyamines and their Analogs in Cancer and other Diseases organization. Thanks are due to the Rector, Prof. Luigi Frati, who allowed the meeting to take place at SAPIENZA University of Rome. I am particularly grateful to all the Authors, mainly to the young scientists, for their scientific support in making this a reality. The Editors also express their gratitude to Professor Gert Lubec, Editor-in-Chief, for the opportunity to publish this special issue in Amino Acids and to Dr. Autar Mattoo for his helpful suggestions. Finally, I am happy to acknowledge the editorial help of my collaborators Drs. Stefania Saccoccio, Giampiero Tempera and Nikenza Viceconte, who assisted me in reading the revised manuscripts that comprise this large volume. The grant support from Italian MIUR (Ministero dell'Istruzione, dell'Università e della Ricerca), Istituto Superiore di Sanità "Project Italy-USA", Istituto Pasteur-Fondazione Cenci Bolognetti and MIUR-PRIN (Cofin) is gratefully acknowledged. Thanks are also due to Fondazione 'Enrico ed Enrica Sovena' for the scholarships given to Nikenza Viceconte and Stefania Saccoccio for supporting their Ph.D.

\section{References}

Agostinelli E, Arancia G, Dalla Vedova L, Belli F, Marra M, Salvi M, Toninello A (2004) The biological functions of polyamine oxidation products by amine oxidases: perspectives of clinical applications. Amino Acids 27:347-358

Agostinelli E, Condello M, Molinari A, Tempera G, Viceconte N, Arancia G (2009) Cytotoxicity of spermine oxidation products to multidrug resistant melanoma cells (M14 ADR2): sensitization by MDL 72527, a lysosomotropic compound. Int $\mathrm{J}$ Oncol 35:485-498

Agostinelli E, Seiler N (2006) Non-irradiation-derived reactive oxygen species (ROS) and cancer: therapeutic implications. Amino Acids 31:341-355

Agostinelli E, Belli F, Molinari A, Condello M, Palmigiani P, DallaVedova L, Marra M, Seiler N, Arancia G (2006) Toxicity of enzymatic oxidation products of spermine to human melanoma cells (M14): sensitization by heat and MDL 72527. Biochim Biophys Acta 1763:1040-1050

Agostinelli E, Marques MPM, Calheiros R, Gil FPSC, Tempera G, Viceconte N, Battaglia V, Grancara S, Toninello A (2010a) Polyamines: fundamental characters in chemistry and biology. Amino Acids 38:393-403

Agostinelli E, Tempera G, Viceconte N, Saccoccio S, Battaglia V, Grancara S, Toninello A, Stevanato R (2010b) Potential anticancer application of polyamine oxidation products formed by amine oxidase: a new therapeutic approach. Amino Acids 38:353-368

Agostinelli E, Toninello A, Vianello F, Stevanato R (2011) Do mammalian amine oxidases and the mitochondrial polyamine transporter have similar protein structures? Amino Acids (in this issue). doi:10.1007/s00726-011-0988-x

Amendola R, Cervelli M, Fratini E, Polticelli F, Sallustio DE, Mariottini P (2009) Spermine metabolism and anticancer therapy. Current Cancer Drug Targets 9:118-130

Arancia G, Calcabrini A, Marra M, Crateri P, Artico M, Martone A, Martelli F, Agostinelli E (2004) Mitochondrial alterations induced by serum amine oxidase and spermine on human multidrug resistant tumor cells. Amino Acids 26:273-282
Arisan ED, Çoker A, Palavan-Ünsal N (2011) Polyamine depletion enhances the roscovitine-induced apoptosis through the activation of mitochondria in HCT116 colon carcinoma cells. Amino Acids (in this issue). doi:10.1007/s00726-011-1040-x

Averill-Bates DA, Cherif A, Agostinelli E, Tanel A, Fortier G (2005) Anti-tumoral effect of native and immobilized bovine serum amine oxidase in a mouse melanoma model. Biochem Pharmacol 69:1693-1704

Bachrach U (2004) Polyamines and cancer: minireview article. Amino Acids 4:307-309

Bachrach U (2011) Concluding remarks. Amino Acids (in this issue). doi:10.1007/s00726-011-1022-Z

Bachrach U, Wang YC, Tabib A (2001) Polyamines: new cues in cellular signal transduction. News Physiol Sci 16:106-109

Baiocco P, Ilari A, Ceci P, Orsini S, Gramiccia M, Di Muccio T, Colotti G (2011) Inhibitory effect of silver nanoparticles on trypanothione reductase activity and Leishmania infantum proliferation. ACS Med Chem Lett 2:230-233

Bandino A, Battaglia V, Bravoco V, Busletta C, Compagnone A, Cravanzola C, Meli F, Agostinelli E, Parola M, Colombatto S (2011) Chronic exposure to agmatine results in the selection of agmatine-resistant hepatoma cells. Amino Acids (in this issue). doi:10.1007/s00726-011-0993-0

Battaglia V, Tibaldi E, Grancara S, Zonta F, Brunati AM, Martinis P, Bragadin M, Grillo MA, Tempera G, Agostinelli E, Toninello A (2011) Effect of peroxides on spermine transport in rat brain and liver mitochondria. Amino Acids (in this issue). doi:10.1007/ s00726-011-0990-3

Battaglia V, Grancara S, Mancon M, Cravanzola C, Colombatto S, Grillo MA, Tempera G, Agostinelli E, Toninello A (2010) Agmatine transport in brain mitochondria: a different mechanism from that in liver mitochondria. Amino Acids 38:423-430

Bonaiuto E, Minarini A, Tumiatti V, Milelli A, Lunelli M, Pegoraro M, Rizzoli V, Di Paolo ML (2011) Synthetic polyamines as potential amine oxidase inhibitors: a preliminary study. Amino Acids (in this issue). doi:10.1007/s00726-011-1006-Z

Brückner H, Flassig S, Kirschbaum J (2011) Determination of biogenic amines in infusions of tea (Camellia sinensis) by HPLC after derivatization with 9-fluorenylmethoxycarbonyl chloride (Fmoc-Cl). Amino Acids (in this issue). doi:10.1007/s00726011-1003-2

Büttner K, Hertel TC, Pietzsch M (2011) Increased thermostability of microbial transglutaminase by combination of several hot spots evolved by random and saturation mutagenesis. Amino Acids (in this issue). doi:10.1007/s00726-011-1015-y

Caccamo D, Currò M, Ferlazzo N, Condello S, Ientile R (2011) Monitoring of transglutaminase 2 under different oxidative stress conditions. Amino Acids (in this issue). doi:10.1007/s00726011-1018-8

Calcabrini A, Arancia G, Marra M, Crateri P, Befani O, Martone A, Agostinelli E (2002) Enzymatic oxidation products of spermine induce greater cytotoxic effects on human multidrug- resistant colon carcinoma cells (LoVo) than on their wild type counterparts. Int J Cancer 99:43-52

Caputo I, Barone MV, Martucciello S, Lepretti M, Esposito C (2009) Tissue transglutaminase in celiac disease: role of autoantibodies. Amino Acids 36:693-699

Casero RA Jr, Pegg AE (2010) Polyamine catabolism and disease. Biochem J 421:323-338

Casero RA, Woster PM (2009) Recent advances in the development of polyamine analogues as antitumor agents. J Med Chem 52:4551-4573

Cecchi L, D’Amato G, Ayres JG, Galan C, Forastiere F, Forsberg B, Gerritsen J, Nunes C, Behrendt H, Akdis C, Dahl R, AnnesiMaesano I (2010) Projections of the effects of climate change on 
allergic asthma: the contribution of aerobiology. Allergy 65: 1073-1081

Cerrada-Gimenez M, Häkkinen MR, Vepsäläinen J, Auriola S, Alhonen L, Keinänen TA (2011) Polyamine flux analysis by determination of heavy isotope incorporation from ${ }^{13} \mathrm{C},{ }^{15} \mathrm{~N}$ enriched amino acids into polyamines by LC-MS/MS. Amino Acids (in this issue). doi:10.1007/s00726-011-1024-x

Cerrada-Gimenez M, Häyrinen J, Juutinen S, Reponen T, Jänne J, Alhonen L (2010) Proteomic analysis of livers from a transgenic mouse line with activated polyamine catabolism. Amino Acids 38:613-622

Cervelli M, Amendola R, Polticelli F, Mariottini P (2011) Spermine oxidase: ten years after. Amino Acids (in this issue). doi: 10.1007/s00726-011-1014-z

Cetrullo S, Facchini A, Stanic I, Tantini B, Pignatti C, Caldarera CM, Flamigni F (2010) Difluoromethylornithine inhibits hypertrophic, pro-fibrotic and pro-apoptotic actions of aldosterone in cardiac cells. Amino Acids 38:525-531

Chaturvedi R, Asim M, Hoge S, Lewis ND, Singh K, Barry DP, de Sablet T, Piazuelo MB, Sarvaria AR, Cheng Y, Closs EI, Casero RA, Jr., Gobert AP, Wilson KT (2010) Polyamines impair immunity to Helicobacter pylori by inhibiting L-arginine uptake required for nitric oxide production. Gastroenterology 139:1686-1698, e1681-e1686

Chaturvedi R, de Sablet T, Coburn LA, Gobert AP, Wilson KT (2011) Arginine and polyamines in Helicobacter pylori-induced immune dysregulation and gastric carcinogenesis. Amino Acids (in this issue). doi:10.1007/s00726-011-1038-4

Childs AC, Mehta DJ, Gerner EW (2003) Polyamine-dependent gene expression. Cell Mol Life Sci 60:1394-1406

Cohen SS (1998) A guide to the polyamines. Oxford University Press, New York

Colotti G, Ilari A (2011) Polyamine metabolism in Leishmania: from arginine to trypanothione. Amino Acids 40:269-285

Compagnone A, Bandino A, Meli F, Bravoco V, Cravanzola C, Parola M, Colombatto S (2011) Polyamines modulate epithelialto-mesenchymal transition. Amino Acids (in this issue). doi: 10.1007/s00726-011-0995-y

Condello S, Currò M, Ferlazzo N, Caccamo D, Satriano J, Ientile R (2011a) Agmatine effects on mitochondrial membrane potential and NF- $\kappa \mathrm{B}$ activation protect against rotenone-induced cell damage in human neuronal-like SH-SY5Y cells. J Neurochem 116:67-75

Condello S, Calabrò E, Caccamo D, Currò M, Ferlazzo N, Satriano J, Magazù S, Ientile R (2011b) Protective effects of agmatine in rotenone-induced damage of human SH-SY5Y neuroblastoma cells: Fourier transform infrared spectroscopy analysis in a model of Parkinson's disease. Amino Acids (in this issue). doi: 10.1007/s00726-011-0994-z

Correa-Fiz F, Reyes-Palomares A, Fajardo I, Melgarejo E, Gutierrez A, García-Ranea JA, Medina MA, Sánchez-Jiménez F (2011) Regulatory cross-talk of mouse liver polyamine and methionine metabolic pathways: a systemic approach to its physiopathological consequences. Amino Acids (in this issue). doi:10.1007/ s00726-011-1044-6

Deasey S, Grichenko O, Du S, Nurminskaya M (2011) Characterization of the transglutaminase gene family in zebrafish and in vivo analysis of transglutaminase-dependent bone mineralization. Amino Acids (in this issue). doi:10.1007/s00726-011-1021-0

Del Duca S, Cai G, Di Sandro A, Serafini-Fracassini D (2010) Compatible and self-incompatible pollination in Pyrus communis displays different polyamine levels and transglutaminase activity. Amino Acids 38:659-667

Di Pierro P, Mariniello L, Sorrentino A, Villalonga R, Chico B, Porta R (2010) Putrescine-polysaccharide conjugates as transglutaminase substrates and their possible use in producing crosslinked films. Amino Acids 38:669-675

Dias CAO, Borges Gregio AP, Rossi D, Carrilho Galvão F, Watanabe TF, Park MH, Valentini SR, Zanelli CF (2011) eIF5A interacts functionally with eEF2. Amino Acids (in this issue). doi: 10.1007/s00726-011-0985-0

Dulloo I, Gopalan G, Melino G, Sabapathy K (2010) The antiapoptotic DeltaNp73 is degraded in a c-Jun dependent manner upon genotoxic stress through the antizyme-mediated pathway. Proc Natl Acad Sci USA 107:4902-4907

Eisenberg T, Knauer H, Schauer A, Buttner S, Ruckenstuhl C, Carmona-Gutierrez D, Ring J, Schroeder S, Magnes C, Antonacci L, Fussi H, Deszcz L, Hartl R, Schraml E, Criollo A, Megalou E, Weiskopf D, Laun P, Heeren G, Breitenbach M, Grubeck-Loebenstein B, Herker E, Fahrenkrog B, Frohlich KU, Sinner F, Tavernarakis N, Minois N, Kroemer G, Madeo F (2009) Induction of autophagy by spermidine promotes longevity. Nat Cell Biol 11:1305-1314

Facchini A, Borzì RM, Olivotto E, Platano D, Pagani S, Cetrullo S, Flamigni F (2011) Role of polyamines in hypertrophy and terminal differentiation of osteoarthritic chondrocytes. Amino Acids (in this issue). doi:10.1007/s00726-011-1041-9

Fincato P, Moschou PN, Spedaletti V, Tavazza R, Angelini R, Federico R, Roubelakis-Angelakis KA, Tavladoraki P (2011a) Functional diversity inside the Arabidopsis polyamine oxidase gene family. J Exp Bot 62:1155-1168

Fincato P, Moschou PN, Ahou A, Angelini R, Roubelakis-Angelakis KA, Federico R, Tavladoraki P (2011b) The members of Arabidopsis thaliana PAO gene family exhibit distinct tissueand organ-specific expression pattern during seedling growth and flower development. Amino Acids (in this issue). doi:10.1007/ s00726-011-0999-7

Fingrut O, Flescher E (2002) Plant stress hormones suppress the proliferation and induce apoptosis in human cancer cells. Leukemia 16:608-616

Folk JE (1983) Mechanism and basis for specificity of transglutaminase-catalyzed epsilon-(gamma-glutamyl) lysine bond formation. Adv Enzymol Relat Areas Mol Biol 54:1-56

Fraser AV, Goodwin AC, Hacker-Prietz A, Sugar E, Woster PM, Casero Jr. RA (2011) Knockdown of Ornithine Decarboxylase Antizyme 1 causes loss of uptake regulation leading to increased N1, N11-bis(ethyl)norspermine (BENSpm) accumulation and toxicity in NCI H157 lung cancer cells. Amino Acids (in this issue). doi:10.1007/s00726-011-1030-z

Gentile A, Antognoni F, Iorio RA, Distefano G, Las Casas G, La Malfa S, Serafini-Fracassini D, Del Duca S (2011) Polyamines and transglutaminase activity are involved in compatible and self-incompatible pollination of Citrus grandis. Amino Acids (in this issue). doi:10.1007/s00726-011-1017-9

Giordano E, Hillary RA, Vary TC, Pegg AE, Sumner AD, Caldarera CM, Zhang X-Q, Song J, Wang JF, Cheung JY, Shantz LM (2011) Overexpression of ornithine decarboxylase decreases ventricular systolic function during induction of cardiac hypertrophy. Amino Acids (in this issue). doi:10.1007/s00726-011-1023-y

Goldenthal MJ, Marín-García J (2004) Mitochondrial signaling pathways: a receiver/integrator organelle. Mol Cell Biochem 262:1-16

Goodwin A, Jadallah S, Toubaji A, Lecksell K, Hicks J, Kowalski J, Bova G, De Marzo A, Netto G, Casero RA Jr (2008) Increased spermine oxidase expression in human prostate cancer and prostatic intraepithelial neoplasia tissues. Prostate 68:766-772

Grancara S, Battaglia V, Martinis P, Viceconte N, Agostinelli E, Toninello A, Deana R (2011) Mitochondrial oxidative stress induced by $\mathrm{Ca}^{2+}$ and monoamines. Different behaviour of liver and brain mitochondria in undergoing permeability transition. Amino Acids (in this issue). doi:10.1007/s00726-011-0991-2 
Griffin M, Casadio R, Bergamini CM (2002) Transglutaminases: nature's biological glues. Biochem J 368:377-396

Groppa MD, Rosales EP, Iannone MF, Benavides MP (2008) Nitric oxide, polyamines and $\mathrm{Cd}$-induced phytotoxicity in wheat roots. Phytochemistry 69:2609-2615

Handa AK, Mattoo AK (2010) Differential and functional interactions emphasize the multiple roles of polyamines in plants. Plant Physiol Biochem 48:540-546

Hauber J (2010) Revisiting an old acquaintance: role for eIF5A in diabetes. J Clin Invest 120:1806-1868

Hegardt C, Johannsson OT, Oredsson SM (2002) Rapid caspasedependent cell death in cultured human breast cancer cells induced by the polyamine analogue N1, N11-diethylnorspermine. Eur J Biochem 269:1033-1039

Hyvönen MT, Uimari A, Vepsäläinen J, Khomutov AR, Keinänen TA, Alhonen L (2011a) Tissue-specific alternative splicing of spermidine/spermine N1-acetyltransferase. Amino Acids (in this issue). doi:10.1007/s00726-011-1027-7

Hyvönen MT, Keinänen TA, Khomutov M, Simonian A, Vepsäläinen J, Park JH, Khomutov AR, Alhonen L, Park MH (2011b) Effects of novel C-methylated spermidine analogs on cell growth via hypusination of eukaryotic translation initiation factor 5A. Amino Acids (in this issue). doi:10.1007/s00726-011-0984-1

Häkkinen MR (2011) Polyamine analysis by LC-MS. Methods Mol Biol 720:505-518

Igarashi K, Kashiwagi K (2010) Modulation of cellular function by polyamines. Int J Biochem Cell Biol 42:39-51

Iismaa SE, Mearns BM, Lorand L, Graham RM (2009) Transglutaminases and disease: lessons from genetically engineered mouse models and inherited disorders. Physiol Rev 89:991-1023

Ikeguchi Y, Bewley MC, Pegg AE (2006) Aminopropyltransferases: function, structure and genetics. J Biochem 139:1-9

Ilari A, Baiocco P, Messori L, Fiorillo A, Boffi A, Gramiccia M, Di Muccio T, Colotti G (2011) A gold-containing drug against parasitic polyamine metabolism: the X-ray structure of Trypanothione Reductase from Leishmania infantum in complex with Auranofin reveals a dual mechanism of enzyme inhibition. Amino Acids (in this issue). doi:10.1007/s00726-011-0997-9

Illingworth C, Michael AJ (2011) Plant ornithine decarboxylase is not post-transcriptionally feedback regulated by polyamines but can interact with a cytosolic ribosomal protein S15 polypeptide. Amino Acids (in this issue). doi:10.1007/s00726-011-1029-5

Iorio RA, Di Sandro A, Paris R, Pagliarani G, Tartarini S, Ricci G, Serafini Fracassini D, Verderio E, Del Duca S (2011) Simulated environmental criticalities affect transglutaminase of Malus and Corylus pollens having different allergenic potential. Amino Acids (in this issue). doi:10.1007/s00726-011-1043-7

Ishii I, Ikeguchi Y, Mano H, Wada M, Pegg AE, Shirahata A (2011a) Polyamine metabolism is involved in adipogenesis of 3T3-L1 cells. Amino Acids (in this issue). doi:10.1007/s00726-0111037-5

Ishii I, Suzuki T, Kaneko H, Uchida M, Suzuki Y, Higashi K, Yagi S, Ariyoshi N, Igarashi K, Kitada M (2011b) Correlation between antizyme 1 and differentiation of vascular smooth muscle cells cultured in honeycomb-like type-I collagen matrix. Amino Acids (in this issue). doi:10.1007/s00726-011-1034-8

Johansson VM, Thuvesson I, Alm K, Oredsson SM (2011) Novel anti-apoptotic effect of the retinoblastoma protein-implications for polyamine analogue toxicity. Amino Acids (in this issue). doi:10.1007/s00726-011-1007-y

Kahana C (2007) Ubiquitin dependent and independent protein degradation in the regulation of cellular polyamines. Amino Acids 33:225-230

Kahana C (2009) Antizyme and antizyme inhibitor, a regulatory tango. Cell Mol Life Sci 66:2479-2488
Kaiser A (2011) Translational control of eIF5a in various diseases. Amino Acids (in this issue). doi:10.1007/s00726-011-1042-8

Kalluri R, Weinberg RA (2009) The basics of epithelial-mesenchymal transition. J Clin Invest 119:1420-1428

Kashiwagi K, Kuraishi A, Tomitori H, Igarashi A, Nishimura K, Shirahata A, Igarashi K (2000) Identification of the putrescine recognition site on polyamine transport protein PotE. J Biol Chem 275:36007-36012

Kashiwagi K, Shibuya S, Tomitori H, Kuraishi A, Igarashi K (1997) Excretion and uptake of putrescine by the PotE protein in Escherichia coli. J Biol Chem 272:6318-6323

Kausch KD, Sobolev AP, Goyal RK, Fatima T, Laila-Beevi R, Saftner RA, Handa AK, Mattoo AK (2011) Methyl jasmonate deficiency alters cellular metabolome, including the aminome of tomato (Solanum lycopersicum L.) fruit. Amino Acids (in this issue). doi:10.1007/s00726-011-1000-5

Keren-Paz A, Bercovich Z, Porat Z, Erez O, Brener O, Kahana C (2006) Overexpression of antizyme-inhibitor in NIH3T3 fibroblasts provides growth advantage through neutralization of antizyme functions. Oncogene 25:5163-5172

Kirschbaum J, Rebscher K, Brückner H (2000) Liquid chromatographic determination of biogenic amines in fermented foods after derivatization with 3, 5-dinitrobenzoyl chloride. J Chromatogr A 881:517-530

Kleman JP, Aeschlimann D, Paulsson M, Van der Rest M (1995) Transglutaminase-catalyzed cross-linking of fibrils of collagen $\mathrm{V} / \mathrm{XI}$ in A204 rhabdomyosarcoma cells. Biochemistry 34: 13768-13775

Koponen T, Cerrada-Gimenez M, Pirinen E, Hohtola E, Paananen J, Vuohelainen S, Tusa M, Pirnes-Karhu S, Heikkinen S, Virkamäki A, Uimari A, Alhonen L, Laakso M (2011) The activation of hepatic and muscle polyamine catabolism improves glucose homeostasis. Amino Acids (in this issue). doi: 10.1007/s00726-011-1013-0

Kramer DL, Diegelman P, Jell J, Vujcic S, Merali S, Porter CW (2008) Polyamine acetylation modulates polyamine metabolic flux, a prelude to broader metabolic consequences. J Biol Chem 283:4241-4251

Kusano T, Berberich T, Tateda C, Takahashi Y (2008) Polyamines: essential factors for growth and survival. Planta 228:367-381

Lewis ND, Asim M, Barry DP, de Sablet T, Singh K, Piazuelo MB, Gobert AP, Chaturvedi R, Wilson KT (2011) Immune evasion by Helicobacter pylori is mediated by induction of macrophage arginase II. J Immunol 186:3632-3641

Li G, Regunathan S, Barrow CJ, Eshraghi J, Cooper R, Reis DJ (1994) Agmatine: an endogenous clonidine-displacing substance in the brain. Science 263:966-969

Lindfors K, Kaukinen K, Mäki M (2009) A role for anti-transglutaminase 2 autoantibodies in the pathogenesis of coeliac disease? Amino Acids 36:685-691

Lindsay GS, Wallace HM (1999) Changes in polyamine catabolism in HL-60 human promyelogenous leukaemic cells in response to etoposide-induced apoptosis. Biochem J 337:83-87

López-Contreras AJ, Ramos-Molina B, Cremades A, Peñafiel R (2010) Antizyme inhibitor 2: molecular, cellular and physiological aspects. Amino Acids 38:603-611

Mangold U (2006) Antizyme inhibitor: mysterious modulator of cell proliferation. Cell Mol Life Sci 63:2095-2101

Martinis P, Battaglia V, Grancara S, Dalla Via L, Di Noto V, Saccoccio S, Agostinelli E, Bragadin M, Grillo MA, Toninello A (2011) Further characterization of agmatine binding to mitochondrial membranes. Involvement of imidazoline $\mathrm{I}_{2}$ receptor. Amino Acids (in this issue). doi:10.1007/s00726-011-0992-1

Marton LJ, Pegg AE (1995) Polyamines as targets for therapeutic intervention. Annu Rev Pharmacol Toxicol 35:55-91 
Marverti G, Guaitoli G, Ligabue A, Frassineti C, Monti MG, Lombardi P, Costi MP (2011) Distamycin A and derivatives as synergic drugs in cisplatin-sensitive and -resistant ovarian cancer cells. Amino Acids (in this issue). doi:10.1007/s00726-0111039-3

Marverti G, Ligabue A, Guerrieri D, Paglietti G, Piras S, Costi MP, Farina D, Frassineti C, Monti MG, Moruzzi MS (2010) Spermidine/Spermine N1-acetyltranferase modulation by novel folate cycle inhibitors in cisplatin-sensitive and -resistant human ovarian cancer cell lines. Gynecol Oncol 117:202-210

Mattoo AK, Handa AK (2008) Higher polyamines restore and enhance metabolic memory in ripening fruit. Plant Sci 174:386-393

Mattoo AK, Minocha SC, Minocha R, Handa AK (2010) Polyamines and cellular metabolism in plants: transgenic approaches reveal different responses to diamine putrescine versus higher polyamines spermidine and spermine. Amino Acids 38:405-413

Minarini A, Milelli A, Tumiatti V, Rosini M, Bolognesi ML, Melchiorre C (2010) Synthetic polyamines: an overview of their multiple biological activities. Amino Acids 38:383-392

Mishina T, Fuchimukai K, Igarashi K, Tashiro K, Shiokawa K (2011) Modification of secondary head-forming activity of microinjected $\Delta \beta$-catenin mRNA by co-injected spermine and spermidine in Xenopus early embryos. Amino Acids (in this issue). doi: 10.1007/s00726-011-0996-x

Morgan DML (1988) Polyamine oxidases and oxidized polyamines. In: Bachrach U, Heimer Y (eds) The physiology of polyamines (Vol. 1). CRC Press, Boca Raton, pp 203-229

Moschou PN, Paschalidis KA, Roubelakis-Angelakis KA (2008) Plant polyamine catabolism. The state of the art. Plant Signal Behav 3:1061-1066

Nayvelt I, John S, Hsu H-C, Yang P, Liu W, Das G, Hyvonen M, Alhonen L, Keinanen T, Shirahata A, Patel R, Thomas T, Thomas TJ (2011) A potential estrogen mimetic effect of a bis(ethyl)polyamine analogue on estrogen receptor positive MCF-7 breast cancer cells. Amino Acids (in this issue). doi: 10.1007/s00726-011-1005-0

Nishimura K, Lee SB, Park JH, Park MH (2011) Essential role of eIF5A-1 and deoxyhypusine synthase in mouse embryonic development. Amino Acids (in this issue). doi:10.1007/s00726011-0986-z

Nurminskaya M, Magee C, Faverman L, Linsenmayer TF (2003) Chondrocyte-derived transglutaminase promotes maturation of preosteoblasts in periosteal bone. Dev Biol 263:139-152

Olsen RR, Chung I, Zetter BR (2011) Knockdown of antizyme inhibitor decreases prostate tumor growth in vivo. Amino Acids (in this issue). doi:10.1007/s00726-011-1032-x

Ono Y, Wook Kim D, Watanabe K, Sasaki A, Niitsu M, Berberich T, Kusano T, Takahashi $\mathrm{Y}(2011)$ Constitutively and highly expressed Oryza sativa polyamine oxidases localize in peroxisomes and catalyze polyamine back conversion. Amino Acids (in this issue). doi:10.1007/s00726-011-1002-3

Park MH (2006) The post-translational synthesis of a polyaminederived amino acid, hypusine, in the eukaryotic translation initiation factor 5A (eIF5A). J Biochem 139:161-169

Park MH, Nishimura K, Zanelli CF, Valentini SR (2010) Functional significance of eIF5A and its hypusine modification in eukaryotes. Review. Amino Acids 38:491-500

Pegg AE (1988) Polyamine metabolism and its importance in neoplastic growth and a target for chemotherapy. Cancer Res 48:759-774

Pegg AE (2008) Spermidine/spermine-N(1)-acetyltransferase: a key metabolic regulator. Am J Physiol Endocrinol Metab 294:E995E1010

Perez-Leal O, Merali S (2011) Regulation of polyamine metabolism by translational control: a mini review. Amino Acids (in this issue). doi:10.1007/s00726-011-1036-6
Pietilä M, Lampinen A, Pellinen R, Alhonen L (2011) Inducible expression of antizyme 1 in prostate cancer cell lines after lentivirus mediated gene transfer. Amino Acids (in this issue). doi:10.1007/s00726-011-1033-9

Pignatti C, Tantini B, Stefanelli C, Flamigni F (2004) Signal transduction pathways linking polyamines to apoptosis. Amino Acids 27:359-365

Pirnes-Karhu S, Sironen R, Alhonen L, Uimari A (2011) Lipopolysaccharide-induced anti-inflammatory acute phase response is enhanced in spermidine/spermine N1-acetyltransferase (SSAT) overexpressing mice. Amino Acids (in this issue). doi: 10.1007/s00726-011-1026-8

Poulin R, Casero RA, Soulet D (2011) Recent advances in the molecular biology of metazoan polyamine transport. Amino Acids (in this issue). doi:10.1007/s00726-011-0987-y

Ramos-Molina B, López-Contreras AJ, Cremades A, Peñafiel R (2011) Differential expression of ornithine decarboxylase antizyme inhibitors and antizymes in rodent tissues and human cell lines. Amino Acids (in this issue). doi:10.1007/s00726-011-1031-y

Reis DJ, Regunathan S (2000) Is agmatine a novel neurotransmitter in brain? Trends Pharmacol Sci 21:187-193

Reyes-Palomares A, Montañez R, Sánchez-Jiménez F, Medina MÁ (2011) A combined model of hepatic polyamine and sulfur amino acid metabolism to analyze S-adenosyl methionine availability. Amino Acids (in this issue). doi:10.1007/s00726-011-1035-7

Rodríguez-Caso C, Montañez R, Cascante M, Sánchez-Jiménez F, Medina MA (2006) Mathematical modeling of polyamine metabolism in mammals. J Biol Chem 281:21799-21812

Rosales EP, Iannone MF, Groppa MD, Benavides MP (2011a) Nitric oxide inhibits nitrate reductase activity in wheat leaves. Plant Physiol Biochem 49:124-130

Rosales EP, Iannone MF, Groppa MD, Benavides MP (2011b) Polyamines modulate nitrate reductase activity in wheat leaves. Involvement of nitric oxide. Amino Acids (in this issue). doi: 10.1007/s00726-011-1001-4

Saiki R, Park H, Ishii I, Yoshida M, Nishimura K, Toida T, Tatsukawa H, Kojima S, Ikeguchi Y, Pegg AE, Kashiwagi K, Igarashi K (2011) Brain infarction correlates more closely with acrolein than with reactive oxygen species. Biochem Biophys Res Commun 404:1044-1049

Satriano J, Matsufuji S, Murakami Y, Lortie MJ, Schwartz D, Kelly CJ, Hayashi S, Blantz RC (1998) Agmatine suppresses proliferation by frameshift induction of antizyme and attenuation of cellular polyamine levels. J Biol Chem 273:15313-15316

Seiler N (1992) Polyamine catabolism and elimination by the vertebrate organism. In: Dowling RH, Fölsch UR, Löser G (eds) Polyamines in the gastrointestinal tract. Kluwer, Dordrecht, pp 65-85

Seiler N, Raul F (2005) Polyamines and apoptosis. J Cell Mol Med 9:623-642

Serafini-Fracassini D, Della Mea M, Tasco G, Casadio R, Del Duca S (2009) Plant and animal transglutaminases: do similar functions imply similar structures? Amino Acids 36:643-657

Shanmugasundaram S, Logan-Mauney S, Burgos K, Nurminskaya M (2011) Tissue transglutaminase regulates chondrogenesis in mesenchymal stem cells on collagen type XI matrices. Amino Acids (in this issue). doi:10.1007/s00726-011-1019-7

Shantz LM, Giordano E (2006) Polyamine metabolism and the hypertrophic heart. In: Wang J-Y, Casero RA (eds) Polyamine cell signaling: physiology, pharmacology and cancer research. Humana Press, Totowa. ISBN 1-58829-625-3 123-137

Shao L, Majumdar R, Minocha SC (2011) Profiling the aminopropyltransferases in plants: their structure, expression and manipulation. Amino Acids (in this issue). doi:10.1007/s00726011-0998-8

Shi C, Welsh PA, Sass-Kuhn S, Wang X, McCloskey DE, Pegg AE, Feith DJ (2011) Characterization of transgenic mice with 
overexpression of spermidine synthase. Amino Acids (in this issue). doi:10.1007/s00726-011-1028-6

Soksawatmaekhin W, Uemura T, Fukiwake N, Kashiwagi K, Igarashi K (2006) Identification of the cadaverine recognition site on the cadaverine-lysine antiporter CadB. J Biol Chem 281:29213-29220

Sollid LM, Khosla C (2005) Future therapeutic options for celiac disease. Nat Clin Pract Gastroenterol Hepatol 2:140-147

Sommer C, Hertel TC, Schmelzer CEH, Pietzsch M (2011) Investigations on the activation of recombinant microbial pro-transglutaminase: in contrast to proteinase $\mathrm{K}$, dispase removes the Histidine-tag. Amino Acids (in this issue). doi:10.1007/s00726011-1016-X

Stevanato R, Mondovì B, Befani O, Scarpa M, Rigo A (1994) Electrostatic control of oxidative deamination catalysed by bovine serum amine oxidase. Biochem J 299:317-320

Tabolacci C, Lentini A, Provenzano B, Beninati S (2011) Evidences for a role of protein cross-links in transglutaminase-related disease. Amino Acids (in this issue). doi:10.1007/s00726-011-1011-2

Tabor H, Rosenthal SM, Tabor CW (1958) The biosynthesis of spermidine and spermine from putrescine and methionine. J Biol Chem 233:907-914

Tavladoraki P, Cona A, Federico R, Tempera G, Viceconte N, Saccoccio S, Battaglia V, Toninello A, Agostinelli E (2011) Polyamine catabolism: target for anti-proliferative therapies in animals and stress tolerance strategies in plants. Amino Acids (in this issue). doi:10.1007/s00726-011-1012-1

Tavladoraki P, Rossi MN, Saccuti G, Perez-Amador MA, Polticelli F, Angelini R, Federico R (2006) Heterologous expression and biochemical characterization of a polyamine oxidase from Arabidopsis involved in polyamine back-conversion. Plant Physiol 141:1519-1532

Teesalu K, Panarina M, Uibo O, Uibo R, Utt M (2011) Autoantibodies from patients with celiac disease inhibit transglutaminase 2 binding to heparin/heparan sulphate and interfere with intestinal epithelial cell adhesion. Amino Acids (in this issue). doi:10.1007/s00726-011-1020-1

Thomas T, Thomas TJ (2001) Polyamines in cell growth and cell death: molecular mechanisms and therapeutic applications. Cell Mol Life Sci 58:244-258

Tobias KE, Kahana C (1995) Exposure to ornithine results in excessive accumulation of putrescine and apoptotic cell death in ornithine decarboxylase overproducing mouse myeloma cells. Cell Growth Differ 6:1279-1285

Tomitori H, Kashiwagi K, Igarashi K (2011) Structure and function of polyamine-amino acid antiporters $\mathrm{CadB}$ and PotE in Escherichia coli. Amino Acids (in this issue). doi:10.1007/s00726011-0989-9
Toninello A, Salvi M, Mondovì B (2004) Interaction of biologically active amines with mitochondria and their role in the mitochondrial-mediated pathway of apoptosis. Curr Med Chem 11:2349-2374

Tseng H-C, Tang J-B, Sudhakar Gandhi PS, Luo C-W, Ou C-M, Tseng C-J, Lin H-J, Chen Y-H (2011) Mutual Adaptation between mouse transglutaminase 4 and its native substrates in the formation of copulatory plug. Amino Acids (in this issue). doi:10.1007/s00726-011-1009-9

Uimari A, Merentie M, Sironen R, Pirnes-Karhu S, Peräniemi S, Alhonen L (2011) Overexpression of spermidine/spermine N1acetyltransferase or treatment with N1-N11-diethylnorspermine attenuates the severity of zinc-induced pancreatitis in mouse. Amino Acids (in this issue). doi:10.1007/s00726-011-1025-9

van den Akker J, Schoorl MJ, Bakker EN, VanBavel E (2010) Small artery remodeling: current concepts and questions. J Vasc Res 47:183-202

van den Akker J, van Weert A, Afink G, Bakker ENTP, van der Pol E, Böing AN, Nieuwland R, VanBavel E (2011a) Transglutaminase 2 is secreted from smooth muscle cells by transamidationdependent microparticle formation. Amino Acids (in this issue). doi:10.1007/s00726-011-1010-3

van den Akker J, van Bavel E, van Geel R, Matlung HL, Janssen GMC, van Veelen PA, Boelens WC, De Mey JGR, Bakker EN (2011b) The redox state of transglutaminase 2 controls arterial remodeling. Plos One 6:e23067. doi:10.1371/journal.pone.0023067

Wang Z, Griffin M (2011) TG2 a novel extracellular protein with multiple functions. Amino Acids (in this issue). doi:10.1007/ s00726-011-1008-x

Wang Z, Collighan RJ, Gross SR, Danen EH, Orend G, Telci D, Griffin M (2010) RGD-independent cell adhesion via a tissue transglutaminase-fibronectin matrix promotes fibronectin fibril deposition and requires syndecan-4/2 and alpha5beta1 integrin co-signaling. J Biol Chem 285:40212-40229

Xu J, Wang W, Ludeman M, Cheng K, Hayami T, Lotz JC, Kapila S (2008) Chondrogenic differentiation of human mesenchymal stem cells in 3D alginate gels. Tissue Eng Part A 14:667-680

Zanelli CF, Valentini SR (2007) Is there a role for eIF5A in translation? Review. Amino Acids 33:351-358

Zhu Q, Huang Y, Marton LJ, Woster PM, Davidson NE, Casero RA Jr (2011) Polyamine analogues modulate gene expression by inhibiting Lysine-Specific Demethylase 1 (LSD1) and altering chromatin structure in human breast cancer cells. Amino Acids (in this issue). doi:10.1007/s00726-011-1004-1 